2 DR. RACHEL CHONG (Orcid ID : 0000-0003-1339-4099)

3

4

Article type : Review Article

Corresponding author mail id:- tina.wong.t.1@snec.com.sg

9

\title{
10 Experimental Models of Glaucoma Filtration Surgery
}

Rachel S. Chong, MBBS, PhD a,b,c,d, Jonathan G. Crowston, MBBS, PhD a,b,c, Tina T. Wong, MBBS, PhD a,b,c

aSingapore National Eye Centre, Singapore

bSingapore Eye Research Institute, Singapore

'Duke-NUS Medical School, Singapore

${ }^{\mathrm{d}}$ Genome Institute of Singapore, Agency for Science Technology and Research, Singapore

Corresponding author: Tina Wong, Singapore National Eye Centre, 11 Third Hospital

Avenue, Singapore 168751

\section{Abstract}

Glaucoma filtration surgery plays an important role in achieving intraocular pressure (IOP) reduction in patients who have high IOP despite maximum medical therapy. Pre-clinical experimental models of glaucoma filtration surgery contribute a great deal to our knowledge of the wound healing processes that predispose to scarring and may lead to poor outcomes. However, this research needs to be interpreted in light of the specific study design, animal model and methods used. We review the existing literature addressing various models of experimental glaucoma filtration surgery, discuss the considerations in assessing these models, and describe future steps in evaluating potenti

This is the author manuscript accepted for publication and has undergone full peer review but has not been through the copyediting, typesetting, pagination and proofreading process, which may lead to differences between this version and the Version of Record. Please cite this article as doi: $10.1111 /$ AOS.14485

This article is protected by copyright. All rights reserved 
1 al therapeutics and bleb characteristics that could impact translational research in this field.

\section{Keywords}

4 Glaucoma; glaucoma filtration surgery; trabeculectomy; glaucoma drainage device; animal model; experimental glaucoma filtration surgery; fibrosis; scarring; wound healing

\section{Introduction}

Animal models provide insight into physiological and pathological processes that underlie health and disease. These models contribute valuable scientific information that is able to complement data from in vitro or in silico studies that are unable to investigate contextual problems within complex biological systems, such as the tissue response to surgical intervention or adjunctive treatments. Extensive anatomical studies, genetic and molecular research techniques using experimental platforms based on animal models have enabled us to delve deeper into exploring potential new diagnostic and therapeutic avenues for many important conditions. At the same time, however, it is crucial to evaluate the extent to which biological mechanisms may vary between species and recognize the limitations of projecting results from animal-based studies into clinical use. This review aims to examine the relevance of animal models of ocular wound healing, and how they have contributed to our understanding of the factors that influence the outcome of glaucoma filtration surgery.

Glaucoma is a leading cause of irreversible blindness, with an estimated 111.5 million people who will be affected by this debilitating disease by 2040 (Tham et al. 2014). Attaining low levels of intraocular pressure (IOP) significantly lowers the risk of progressive sight loss in glaucoma patients (Heijl et al. 2002). When IOP remains suboptimally-controlled in spite of maximum tolerable medical therapy, glaucoma filtration surgery may be warranted. Surgical procedures, such as trabeculectomy or implantation of drainage devices, create an artificial outflow shunt and result in the formation of a fluid-filled bleb, due to the collection of aqueous humour under the conjunctiva and Tenon's capsule. Maintenance of a healthy, functioning bleb is key to ensuring long-term surgical success. Bleb function can, however, become compromised by the formation of subconjunctival fibrosis leading to restriction in aqueous outflow and elevation of the IOP (Roth et al. 1991). 
1 Prevention of scarring is an important aspect of maintaining satisfactory IOP reduction 2 following GFS. This is currently achieved with the administration of intensive topical 3 corticosteroids following surgery, as well as the intraoperative application of anti4 proliferative agents such as 5-fluorouracil (5-FU) (Green et al. 2014), anti-vascular endothelial growth factor agents such as bevacizumab (van Bergen et al. 2015; van Bergen et al. 2011) and mitomycin C (MMC) (Bergstrom et al. 1991; Wilkins et al, 2005). 5-FU interferes with transcription, and together with $M M C$, acts through the inhibition of DNA synthesis that influences myofibroblast proliferation and survival respectively resulting in a dampened scarring response. However, the non-specific effects of these drugs can result in complications such as corneal toxicity and thin-walled blebs that may lead to loss of sight due to infections such as endophthalmitis (Greenfield et al. 1996; Higginbotham et al. 1996; Jampel et al. 1992; The Fluorouracil Filtering Surgery Group. 1989; Wells et al. 2003; Wilkins et al. 2005). Furthermore, failure of GFS still occurs in a substantial number of patients who are at higher risk of scar formation, despite the use of anti-proliferative agents (Green et al. 2014). Therefore, the decision to pursue surgical intervention for glaucoma cannot be regarded lightly.

Various studies that examined risk factors and predictors of surgical failure in patients thus far have been based on conjunctival biopsies (Chang et al. 2009), impression cytology, and tear studies examining changes in pro-inflammatory cell populations and secreted cytokines (Chong et al. 2010). These studies have yielded important information about the cellular response and molecular pathways that could predispose bleb failure. However, they are also limited by difficulties with acquiring samples and multiple clinical factors such as the duration and combination of topical eyedrops administered, or differences in surgical technique that may potentially confound the results. The development of robust experimental models of glaucoma filtration surgery has certainly provided additional insight to our understanding of the pathways that contribute to fibrosis and scarring processes underlying bleb failure. This review summarises the various experimental models of glaucoma filtration surgery that have been published thus far and evaluates how they have contributed to our knowledge of the mechanisms underlying bleb stability and conjunctival health during the post-operative recovery process.

\section{Clinical evaluation of glaucoma filtration surgery success}

This article is protected by copyright. All rights reserved 
1 As described by the 'Moorfields Safer Surgery System' (Wells et al. 2003), steps for 2 trabeculectomy include a conjunctival incision, creation of a partial-thickness scleral flap, 3 application of anti-metabolite agents in the conjunctival pocket for a fixed interval followed 4 by copious irrigation, paracentesis, sclerostomy, peripheral iridectomy, suturing of the scleral 5 flap and finally conjunctival closure. Drainage devices comprise silicone tubes that are 6 inserted into the anterior chamber, ciliary sulcus or vitreous chamber via a scleral tunnel, and 7 are connected to a plate implant that is sutured to the sclera and drains into the 8 subconjunctival space at a site posterior to the limbus. Hence blockage occurring at any point 9 along the outflow pathway could lead to surgical failure, although subconjunctival scarring is thought to be the major contributing factor to this (Skuta \& Parrish, 1987).

Clinically relevant markers of surgical success following trabeculectomy or implantation of drainage devices include IOP measurements, assessment of bleb morphological characteristics (Filippopoulos et al. 2009) including bleb area, height and vascularity, as well as the presence of subepithelial microcysts that indicate good drainage. Elevated IOP with the presence of a flat bleb and scarred conjunctiva would necessitate further intervention with medical therapy, suture release or bleb-manipulation procedures with anti-proliferative agents.

It is not fully understood to date, why particular patients may be more prone to failure of filtration surgery than others. Hence, experimental models of glaucoma filtration surgery may provide further insight into the risk factors for bleb failure due to the relative ease of obtaining tissue or aqueous humour samples and the advantage of establishing standardized peri-operative conditions in comparison with clinical studies. This has enabled in-depth quantitative analysis of changes that occur in the cellular, biochemical or genetic composition of affected tissues following experimental glaucoma filtration surgery. The availability of clinical tools that have specially been adapted for animal studies such as slit lamp microscopy, anterior segment optical coherence tomography and in vivo confocal microscopy has further strengthened our ability to measure clinically-relevant markers of success in these models.

3. Considerations when evaluating animal models of glaucoma filtration surgery

\subsection{Surgical techniques}

32 The key surgical steps in trabeculectomy should be closely replicated in the animal model to 33 provide similar post-operative events. This is important for the translation of findings reported in animal models when a therapeutic target is being investigated for a potential 
1 clinical application. The effects of conjunctival dissection have been recapitulated experimentally by induction of conjunctival scarring via subconjunctival injections in mice (Reichel et al. 1998) as well as circumferential conjunctival incisions made along the limbus with or without suturing in mice (Yamanaka et al. 2007) and rabbits (Takeuchi et al. 2010). Modified versions of trabeculectomy have also been performed with or without the creation of a scleral flap, or peripheral iridectomy in rabbits (Ekinci et al. 2014; Memarzadeh et al. 2009; Perkins et al. 2002; Takeuchi et al. 2009), dogs (Kojima et al. 2015) and non-human primate (Ye et al. 2010) species.

It is important to consider that the omission of various steps in experimental glaucoma filtration surgery, such as the creation of a scleral flap or peripheral iridectomy, results in varying degrees of surgical trauma that in turn incites different levels of inflammation in the eye. Therefore, models that describe modified techniques of glaucoma filtration surgery are useful for evaluating tissue responses that are highly specific to that particular model. Techniques that only investigate conjunctival injury in the presence of an intact sclera, for example, do not take into account the effect of the large body of pro-inflammatory cytokines or growth factors such as transforming growth factor- $\beta$, platelet-derived growth factor (Takai et al. 2012) and vascular endothelial growth factor (Li et al. 2009; Lopilly Park et al. 2012), found in the aqueous humour that could affect the surgical outcome. Models of conjunctival scarring alone in the absence of a fluid-filled bleb also do not take into account how the physical characteristics of the subconjunctival bleb may affect aqueous outflow (Fan Gaskin et al. 2014). Other considerations include the composition of the drainage devices used for intra-cameral cannulation, which may be made of polyurethane instead of silicone. These devices possess anti-thrombogenic coatings (Gupta et al. 2007), although it is not known how these attributes could affect the ocular and subconjunctival inflammatory responses. Factors that can influence wound healing in models of experimental glaucoma filtration surgery also include the use of sutures, whether they are absorbable or non-absorbable, as well as the application of post-operative corticosteroid preparations.

30 Rabbits are the most commonly used species in experimental models of glaucoma filtration surgery (Arslan et al. 2012; Cordeiro et al. 1997; Ekinci et al. 2014; Kim et al. 2010; Lukowski et al. 2013; Ma et al. 2014; Perkins et al. 2002; Sen et al. 2010; Soohoo et al. 2012; Takeuchi et al. 2010; Wong et al. 2005; Zhong et al. 2011). The average horizontal cornea diameter of a rabbit is $11.75 \mathrm{~mm}$ (Bozkir et al. 1997), which closely approximates the human 
1 eye. Therefore, it is possible to perform surgery on a rabbit eye using techniques that most closely resemble a complete trabeculectomy in glaucoma patients. Tube surgery has also been described in rabbits using various devices that are in clinical use such as the Molteno (Nguyen et al. 2012), Baerveldt (Lloyd et al. 1996) or Ahmed implant (Li et al. 2009) or modified intravenous cannulae (Lukowski et al. 2013). IOP measurements are typically achieved in rodents using a rebound tonometer specially-designed for use in the laboratory, such as the Icare Tonolab (Icare, Vantaa, Finland). However, one of the major limitations of rabbit models of glaucoma filtration surgery however, is the absence of a significant sustained decrease in intraocular pressure, which is a critical end-point in clinical studies that determine surgical successThis lack of IOP reduction appears to be the case in all animal studies regardless of species, although it has been postulated that the ciliary epithelium and iris may have a greater tendency to extend forward into the anterior chamber to block the surgical fistula in rabbits (Miller et al. 1989; Ma et al. 2014).

Mice are also widely-used due to their availability and comparatively lower cost. Another advantage is that they can theoretically be bred for a specific gene of interest for further evaluation as a therapeutic target, although to date, there is no established genetic model used to study subconjunctival fibrosis. Furthermore, surgery in mice poses greater technical challenges due to the relatively small size of their eyeballs. Therefore, the majority of research published on mouse models of 'glaucoma filtration surgery' are largely concerned with studying the response to conjunctival injury (Cordeiro et al. 1999; Reichel et al. 1998; Seet et al. 2010; Yamanaka et al. 2007) that may be induced using incisions or injections, rather than a true representation of trabeculectomy. Models that incorporate the creation of a sclerostomy e.g. using a $30 \mathrm{G}$ needle to penetrate the anterior chamber, on the other hand, permit research into the effect of aqueous egress at the bleb, including the role of inflammatory cytokines and tissue re-modelling responses. Rat models (Wang et al. 2014; Zhu et al. 2015) have also been described in a few studies, with similar considerations as previously described in mice although their larger eyes result in the additional benefit of providing greater quantities of ocular tissue for sampling, without the need for pooling tissues from several animals in order to perform protein or genetic assays. primates (Gressel et al. 1984; Ye et al. 2010) have also been used to experimentally model glaucoma filtration surgery. These species have larger eyes and hence present fewer issues 
1 with surgical accessibility, but offer limited benefit apart from this. They are also much more expensive to maintain and require specialized facilities, which could result in prohibitive costs for many researchers.

\subsection{Use of models in the evaluation of novel therapeutic platforms}

6 Experimental models of glaucoma filtration surgery have been used to evaluate various systems of drug delivery, as well as different types of therapeutic agents. It is important to consider how the method of drug delivery can affect the wound healing response and bleb stability. Slow-release preparations for example, may result in longer exposure to potentially toxic excipient agents in addition to the extended therapeutic benefits. Frequent subconjunctival injections could also result in an increased predisposition to scarring and fibrosis in the long-term.

A common strategy for the prevention of bleb adhesion and fibrosis, utilizes subconjunctival spacers that are constituted from a broad spectrum of gel-like substances and placed in the subconjunctival space or underneath the scleral flap. These are composed of materials such as chitosan (Zhu et al. 2015), carbopol (Koz et al. 2007), gelatin (Kojima et al. 2015), hyaluronic acid-carboxymethyl cellulose (Takeuchi et al. 2009), poly(L-lactide-co-epsiloncaprolactone) (PLCL) (Okuda et al. 2008), poly(2-hydroxyethyl methacrylate) (P[HEMA]) or poly(lactic-co-glycolic acid) (PLGA) systems (Schoenberg et al. 2015), and may be biodegradable e.g. chitosan, PLCL, PLGA or non-biodegradable e.g. P[HEMA]. Potential benefits of using such devices are that the materials are biocompatible and can be manipulated to form high density drug-complexes that enable stable, controlled drug release over several days to months (Bernkop-Schnurch \& Dunnhaupt, 2012; Hoare \& Kohane, 2008). However, the long-term effects of these substances on ocular health remain to be seen. Results from clinical studies evaluating the safety and efficacy of a biodegradable collagenglycosaminoglycan copolymer matrix spacer (Ologen) used as a surgical adjunct during trabeculectomy over 5 years or more suggest that this implant is safe with similar treatment outcomes with MMC, although these studies involved relatively small numbers of patients (Cillino

al. 2016; Singh

et

al. 2018).

Other drug delivery systems that have been tested also include virus-mediated gene transfer (Heatley et al. 2004; Perkins et al. 2002; Yamanaka et al. 2007), liposomes (Alvarado, 1989; Simmons et al. 1988; Tilleul et al. 1997) and nanoparticles (Butler et al. 2013; Santos et al. 
1 2006; Tilleul et al. 1997; Ye et al. 2010) in combination with antiproliferative/migratory agents or small molecule therapies. These were administered in the form of drug-soaked sponges left in situ for a short duration intra-operatively, injected subconjunctivally or applied in the form of topical eyedrops. The precise mechanism of action of each therapeutic agent that has been described in the literature is outside the scope of this review, and has been elaborated on elsewhere (Fan Gaskin et al. 2014; Fan Gaskin et al. 2014; Khaw et al. 2001;

It is possible that a combination of drug delivery approaches is required to ensure successful inhibition of the scarring response, which is maintained over time. One area of research that has shown recent breakthrough using a combined approach is the subconjunctival application of peglyated anionic lipid-peptide-siRNA nanocomplexes containing RNA molecules that silence transcription factors involved in tissue fibrosis. Small interfering RNAs (siRNAs) are naturally occurring molecules that are thought to exert high specific inhibitory effects on target genes. Lipid-peptide-nanoparticle-mediated siRNA therapy that carries an anionic charge further augments its potency owing to greater cell penetration, higher genetic cargo stability and a prolonged duration of action ${ }^{65}$. Studies on a rabbit model of glaucoma filtration surgery (Fernando et al. 2018; Man et al. 2016) as well as a mouse model of conjunctival scarring (Seet et al. 2018) have demonstrated that these nanocomplexes have impressive effects on dampening the scarring response and bleb survival, and importantly do not appear to result in any systemic or ocular toxicity.

\subsection{Experimental outcome measures - the biological response}

24 Experimental animal models provide the major advantage of producing readily available biological samples to perform multiple assays on. This has enabled the development of studies that make use of objective quantitative methods such as real-time polymerase chain reactions (qPCR) to study genetic alterations in experimental glaucoma filtration surgery, as well as histological research that evaluates differences in inflammatory cell populations in terms of cell proliferation/density, cell toxicity and changes in the extracellular matrix (Chong et al. 2017; Seet et al; 2010). Changes in the inflammatory cell profile mediate scarring predisposition due to the increased secretion of pro-fibrotic cytokines, together with the deposition of extracellular matrix proteins such as collagen and fibronectin that are important modulators of wound healing. 
2 Biological ex vivo assays are complemented by in vivo assessments of clinical relevant markers of surgical outcome, such as bleb morphology and function. This includes the use of slit lamp microscopy photographs to document changes in bleb features such as bleb area, vascularity and height as well as any adverse effect on the ocular surface e.g. corneal toxicity resulting from therapeutic interventions. Anterior segment optical coherence tomography also enables objective measurements of bleb height and bleb wall thickness following surgery. Bleb morphology and vascularity are thought to be indicators of long-term bleb survival, hence several clinical bleb grading systems have been proposed based on these features (Wells et al. 2006). However, there is a lack of evidence that such scoring systems have any impact on surgical outcomes. This may be due in part to their limited ability to evaluate blebs with mixed features, as well as the fact that these assessments are mainly confined to the superficial aspects of bleb appearance. In order to address the question of whether there is adequate flow occurring within the bleb using animal models, in vivo confocal microscopy can be performed to evaluate the presence of conjunctival subepithelial microcysts, since this has been reported to suggest good bleb filtration clinically (Labbe et al. 2005). An example of these imaging methods is shown in Figure 1 (Chong et al. 2017).

Capsular porosity has also been postulated to be a major determinant of fluid outflow (Gardiner et al. 2010), and has been studied in rabbits following the implantation of intracameral drainage devices (Nguyen et al. 2012; Pandav et al. 2016) where the authors found that infusions of balanced salt solution at physiological pressures in the absence of inflammatory cytokines was sufficient to alter the porosity of the fibrous capsule at the surgical site. Thus, there are clearly other important parameters apart from bleb morphology and vascularity that need to be studied in greater detail, such as the effect of shear stress on tissue re-modelling and alterations in functional drainage that occur within the bleb. Structural and functional assessments of retinal ganglion cell health are also often missing from experimental reports of glaucoma filtration surgery, although these are commonly used to monitor glaucoma patients in clinics. Future laboratory-based studies of glaucoma filtration surgery that include measures of retinal ganglion cell function using electrophysiology or structural losses using optical coherence tomography, for example, would no doubt add significant value to research in this area.

\section{Future directions}


1 Much of the research on glaucoma filtration surgery has been focused on alterations in the conjunctiva and Tenon's tissues, since these are major structural components that give rise to the bleb. However, lesser is known about factors that influence drainage away from the subconjunctival space. Since IOP does not demonstrate any appreciable reduction following glaucoma surgery in experimental models despite establishing patent connections between the anterior chamber and subconjunctival space, it may be that maintaining functional distal drainage pathways away from the subconjunctival space is critical in ensuring surgical success. This is particularly relevant with the advent of minimally invasive glaucoma surgery, where animal models may enable more thorough evaluations of different surgical approaches ( $a b$ interno versus $a b$ externo) and the impact of different types of devices on distal outflow pathways (Rodgers et al. 2018). The effect of chronic glaucoma medication on the human ocular surface has been studied in great detail, although these findings have yet to be translated to pre-clinical platforms for further testing. An experimental model of glaucoma filtration surgery that also emulates the effect of chronic exposure to glaucoma drugs and their excipient agents would no doubt add value to our understanding of how the woundhealing response is affected by these treatments.

Newer methods of imaging may provide further insight into the structure, function and regulation of drainage channels such as the use of in vivo two-photon imaging to study collagen second harmonic generation. This has enabled ultra-high resolution studies of the locations of episcleral vessels, intrascleral plexuses, collector channels and Schlemm's canal in mice (Gonzalez et al. 2017). Optical coherence tomography angiography is a promising technology that is gradually gaining traction clinically to quickly and non-invasively assess vessel morphology. Incorporating methods that enable clear visualization of the distal aqueous outflow pathways will undoubtedly be extremely valuable in our understanding of how these systems are altered following glaucoma filtration surgery. Another emerging area of interest is the study of conjunctival lymphatics and whether this system may contribute to satisfactory aqueous drainage from the bleb (Bouhenni et al. 2016; Yu et al. 2009).

Greater efforts at developing models that more closely resemble human disease processes involved in glaucoma, including ageing changes of the conjunctiva and episcleral tissues or the effect of chronic glaucoma medication on wound healing may also provide more accurate depictions of the expected surgical outcome. 
1 Other major considerations in translating the results from animal-based studies to clinical use include issues with dosing and bioavailability. Important differences in the genetic, molecular,

3 biochemical, immunological and anatomical make-up exist across species. Therefore, promising results from a novel therapy with a dosing regimen that works well in a mouse may not demonstrate similar efficacy in larger animals. It is also particularly challenging to interpret results from published studies, when there is often a far greater body of unpublished work addressing important relevant issues e.g. pharmacokinetic testing that remains mostly inaccessible. The CAT-152 Trabeculectomy Study is a notable example of a monoclonal antibody against transforming growth factor- $\beta 2$, a key cytokine that modulates tissue repair, which did not show any difference from placebo in preventing the failure of primary trabeculectomy in a large phase III randomized control trial (CAT-152 0102 Trabeculectomy Study Group. 2007) in spite of promising results from previous studies in both animals and humans. Perhaps more research should be devoted to evaluating how to extrapolate data on the efficiency of drug metabolism, which could potentially affect drug pharmacokinetic properties and ocular bioavailability from rodents or other experimental models to humans (Blais et al. 2017), in order to facilitate greater precision in developing clinically-useful treatments.

\section{Conclusion}

20 Glaucoma filtration surgery is a key aspect of managing IOP in many patients, although our understanding of what constitutes an ideal bleb in clinics is still limited to gross assessments of bleb morphology and vascularity. To date, no targeted anti-scarring treatment has demonstrated significant benefit in clinical studies of glaucoma filtration surgery, apart from broad spectrum anti-proliferative agents such as mitomycin $\mathrm{C}$ and 5-fluorouracil. Therefore, experimental models continue to serve an important role in the pre-clinical evaluation of potential drug candidates as they provide additional insight into the underlying cellular and molecular changes that modulate the wound healing response, which may aid the development of novel anti-scarring strategies. However, this research needs to be carefully interpreted in light of the specific study design, animal model and methodology used. In the continued efforts to identify and develop newer and safer anti-scarring therapies for glaucoma filtering surgeries, it is critical to consider the relevant outcomes from different surgical animal models in order to discern what may be translated to human patients. 


\section{Literature search}

A literature search of PubMed and Google Scholar was conducted in April 2018. Articles published in peer-reviewed journals on experimental models of glaucoma filtration surgery were included. The search used the following key words: experimental model and glaucoma filtration surgery; experimental model and trabeculectomy; experimental model and glaucoma drainage. Full articles or their abstracts that were written in English were included. Studies that were not performed on animal models were excluded from this review.

\section{Disclosure}

The authors report no proprietary or commercial interest in any product mentioned or concept discussed in this article. RC is supported by the Agency for Science, Technology and Research.

\section{References}

Alvarado JA (1989): The use of a liposome-encapsulated 5-fluoroorotate for glaucoma surgery: I. Animal studies. Trans Am Ophthalmol Soc. 87:489-514.

Arslan S, Aydemir O, Guler M, Dagli AF (2012): Modulation of postoperative scarring with tacrolimus and octreotide in experimental glaucoma filtration surgery. Curr Eye Res. 37(3):228-33.

Bergstrom TJ, Wilkinson WS, Skuta GL, et al (1991): The effects of subconjunctival mitomycin C on glaucoma filtration surgery in rabbits. Arch Ophthalmol. 109: 17251730.

Bernkop-Schnurch A, Dunnhaupt S (2012): Chitosan-based drug delivery systems. European journal of pharmaceutics and biopharmaceutics. 81(3):463-9.

Blais EM, Rawls KD, Dougherty BV et al (2017): Reconciled rat and human metabolic networks for comparative toxicogenomics and biomarker predictions. Nature Communications. 8:14250.

Bouhenni RA, Al Jadaan I, Rassavong H et al (2016): Lymphatic and blood vessel density in human conjunctiva after glaucoma filtration surgery. J Glaucoma. 25(1):e35-8.

Bozkir G, Bozkir M, Dogan H, Aycan K, Guler B (1997): Measurements of axial length and radius of corneal curvature in the rabbit eye. Acta Med Okayama. 51(1):9-11. 
Butler MR, Ponce CM, Weinstock YE et al (2013): Topical silver nanoparticles result in improved bleb function by increasing filtration and reducing fibrosis in a rabbit model of filtration surgery. IOVS. 54(7):4982-90.

CAT-152 0102 Trabeculectomy Study Group (2007): A phase III study of subconjunctival human anti-transforming growth factor $\beta 2$ monoclonal antibody (CAT152) to prevent scarring after first-time trabeculectomy. 114:1822-30.

Chang L, Wong T, Ohbayashi M et al (2009): Increased mast cell numbers in the conjunctiva of glaucoma patients: a possible indicator of preoperative glaucoma surgery inflammation. Eye (Lond). 239:1859-65.

Chong RS, Jiang YZ, Boey PY et al (2010): Tear cytokine profile in medicated glaucoma patients: effect of monocyte chemoattractant protein 1 on early post-trabeculectomy outcome. Ophthalmology. 117:2353-8.

Chong RS, Lee YS, Chu SWL, Toh LZ, Wong TTL (2017): Inhibition of monocyte chemoattractant protein 1 prevents conjunctival fibrosis in an experimental model of glaucoma filtration surgery. IOVS. 58:3432-9.

Cillino S, Casuccio A, Di Pace F et al (2016): Biodegradable collagen matrix implant versus mitomycin-C in trabeculectomy: five-year follow-up. BMC Ophthalmol. 16:24

Cordeiro M, Constable P, Alexander R, Bhattacharya S, Khaw PT (1997). Effect of varying the mitomycin-C treatment area in glaucoma filtration surgery in the rabbit. IOVS. 38(8):1639-46.

Cordeiro MF, Reichel MB, Gay JA et al (1999): Transforming growth factor-beta1, beta2, and beta 3 in vivo: effects on normal and mitomycin C-modulated conjunctival scarring. IOVS. 40(9):1975-82.

Ekinci M, Cagatay HH, Ceylan E et al (2014): Reduction of conjunctival fibrosis after trabeculectomy using topical alpha-lipoic acid in rabbit eyes. J Glaucoma. 23(6):372-9.

Fan Gaskin JC, Nguyen DQ, Soon Ang G, O’Connor J, Crowston JG (2014): Wound healing modulation in glaucoma filtration surgery - conventional practices and new perspectives: the role of antifibrotic agents (Part I). J Curr Glaucoma Pract. 8(2):37-45.

Fan Gaskin JC, Nguyen DQ, Soon Ang G, O'Connor J, Crowston JG (2014): Wound healing modulation in glaucoma filtration surgery - conventional practices and new 
perspectives: antivascular endothelial growth factor and novel agents (Part II). J Curr Glaucoma Pract. 8(2):46-53.

Fernando O, Tagalakis A, Awwad S et al (2018): Development of targeted siRNA nanocomplexes to prevent fibrosis in experimental glaucoma filtration surgery. Molecular Therapy. 26(12):2812-22.

Filippopoulos T, Hanna E, Chen TC et al (2009): Correlation of filtration bleb morphology with histology. Internation Ophthalmology Clinics. 49(1):71-82.

Gardiner B, Smith D, Coote M, Crowston J (2010): Computational Modeling of Fluid Flow and Intra-Ocular Pressure following Glaucoma Surgery. PLoS ONE. 5(10):e13178.

Gonzalez JM, Ko MJ, Masedunskas A et al (2017). Toward in vivo two-photon analysis of mouse aqueous outflow structure and function. Experimental Eye Research. 158:16170.

Green E, Wilkins M, Bunce C, et al (2014): 5-Fluorouracil for glaucoma surgery. Cochrane Database Syst Rev. 2: CD001132.

Greenfield DS, Suñer IJ, Miller MP, et al (1996): Endophthalmitis after filtering surgery with mitomycin. Arch Ophthalmol. 114: 943- 949.

Gressel MG, Parrish RK 2nd, Folberg R (1984): 5-fluorouracil and glaucoma filtering surgery: I. An animal model. Ophthamology. 91(4):378-83.

Gupta A, Mehta Y, Juneja R, Trehan N (2007). The effect of cannula material on the incidence of peripheral venous thrombophlebitis. Anaesthesia. 62:1139-42.

Heatley G, Kiland J, Faha B et al (2004). Gene therapy using p21WAF-1/Cip-1 to modulate wound healing after glaucoma trabeculectomy surgery in a primate model of ocular hypertension. Gene ther. 11:949-55.

Heijl A, Leske MC, Bengtsson B et al (2002). Reduction of intraocular pressure and glaucoma progression: results from the Early Manifest Glaucoma Trial. Arch Ophthalmol. 120(10):1268-79.

Higginbotham E, Steven KR, Musch DC, et al (1996): Bleb-related endophthalmitis after trabeculectomy with mitomycin C. Ophthalmology. 103: 650- 656. 
Hoare TR, Kohane DS (2008): Hydrogels in drug delivery: progess and challenges. Polymer. 49980:1993-2007.

Jampel HD, Pasquale LR, DiBernardo C (1992): Hypotonous maculopathy following trabeculectomy with mitomycin C. Arch Ophthalmol. 110: 1049- 1050.

Khaw PT, Chang L, Wong TTL, Mead A, Daniels JT, Cordeiro MF (2001): Modulation of wound healing after glaucoma surgery. Curr Opin in Ophthalmol. 1:143-8.

Kim TH, Oh HC, Yoo YH et al (2010): An experimental study of rabbit conjunctival epithelial toxicity using co-treatment with mitomycin-C and a histone deacetylase inhibitor. Arch Pharm Res. 33(8):1261-7.

Kojima S, Sugiayama T, Takai S et al (2015). Effects of gelatin hydrogel loading Mitomycin $\mathrm{C}$ on conjunctival scarring in a canine filtration surgery model. IOVS. 56:2601-5.

Koz OG, Ozhuy S, Tezel GG et al (2007): The effect of paclitaxel on conjunctival wound healing: a pilot study. J Glaucoma. 16(7):610-5.

Labbe A, Dupas N, Hamard P, et al (2005): In vivo confocal microscopy study of blebs after filtering surgery. Ophthalmology. 112: 1979- 1986.

Li Z, Van Bergen T, Van de Veire S et al (2009): Inhibition of vascular endothelial growth factor reduces scar formation after glaucoma filtration surgery. IOVS. 50:5217-25. Lloyd MA, Baerveldt G, Bguyen QH, Minckler DS (1996): Long-term histologic studies of the Baerveldt implant in a rabbit model. J Glaucoma. 5(5):334-9.

Lopilly Park HY, Kim JH, Ahn MD, Park CK (2012): Levels of vascular endothelial growth factor in tenon tissue and results of glaucoma surgery. Arch Ophthalmol. 130:685-9.

Lukowski ZL, Min J, Beattie AR et al (2013): Prevention of Ocular scarring after glaucoma filtering surgery using the monoclonal antibody LT1009 (Sonepcizumab) in a rabbit model. J Glaucoma. 22(2):145-51.

Ma J, Li X, Zhang W et al (2014): CSM enhances the filtration bleb survival in rabbit model of experimental glaucoma surgery. Curr Eye Res. 39(10):982-8. 
Man CYW, Tagalakis AD, Manunta MD et al (2016): Receptor-targeted liposomepeptide-siRNA nanoparticles represent an efficient delivery system for MRTF silencing in conjunctival fibrosis. Sci Rep. 6:21181.

Maruichi M, Takai S, Sugiyama T et al (2004): Role of chymase on growth of cultured canine Tenon's capsule fibroblasts and scarring in a canine conjunctival flap model. Experimental Eye Research. 79(1):111-8.

Memarzadeh F, Varma R, Lin L et al (2009): Postoperative use of bevacizumab as an antifibrotic agent in glaucoma filtration surgery in the rabbit. IOVS. 50(7):3233-7.

Miller MH, Grierson I, Unger WI, Hitchings RA (1989): Wound healing in an animal model of glaucoma fistulizing surgery in the rabbit. Ophthalmic Surg. 20:350- 357

Nguyen DQ, Ross C, Li YQ et al (2012): A model to measure fluid outflow in rabbit capsules post glaucoma implant surgery. IOVS. 53:6914-9.

Okuda T, Higashide T, Fukuhira Y et al (2008): A thin honeycomb-patterned film as an adhesion barrier in an animal model of glaucoma filtration surgery. J Glaucoma. 18(3):220-6.

Pandav S, Ross C, Thattaruthody F et al (2016). Porosity of bleb capsule declines rapidly with fluid challenge. J Curr Glaucoma Pract. 10(3):91-6.

Perkins TW, Faha B, Ni M et al (2002). Adenovirus-mediated gene therapy using human p21WAF-1/Cip-1 to prevent wound healing in a rabbit model of glaucoma filtration surgery. JAMA Ophthalmology. 120(7):941-9.

Reichel MB, Cordeiro MF, Alexander RA et al (1998): New model of conjunctival scarring in the mouse eye. British Journal of Ophthalmology. 82(9):1072-7.

Rodgers C, Meyer A, Rosenberg N et al (2018): The impact of conjunctival flap method and drainage cannula diameter on bleb survival in the rabbit model. PLoS One. 24(13): 0196968 .

Roth SM, Spaeth GL, Starita RJ, et al (1991): Effects of postoperative corticosteroids on trabeculectomy and the clinical course of glaucoma. The five-year follow up study. Ophthalmic Surg. 22: 724- 729. 
Santos AL, Bochot A, Doyle A, et al (2006): Sustained release of nanosized complexes of polyethylenimine and anti-TGF- $\beta 2$ oligonucleotide improves the outcome of glaucoma surgery. J Control Release. 112:369-81.

Schoenberg ED, Blake DA, Swann FB et al (2015): Effect of two novel sustained-release drug delivery systems on bleb fibrosis: an in vivo glaucoma drainage device study in a rabbit model. Transl Vis Sci Technol. 4(3):4

Seet LF, Su R, Barathi VA et al (2010): SPARC deficiency results in improved surgical survival in a novel mouse model of glaucoma filtration surgery. PloS One. 5:e9415.

Seet L, Tan Y, Toh L et al (2018): Targeted therapy for the post-operative conjunctiva: SPARC silencing reduces collagen deposition. Br J Ophthalmol. 102(10):1460-70.

Sen E, Balikoglu-Yilmaz M, Bozdag-Pehlivan S et al (2010): Effect of doxycycline on postoperative scarring after trabeculectomy in an experimental rabbit model. Journal of Ocular Pharmacology and Therapeutics. 26(5):399-406.)

Simmons ST, Sherwood MB, Nichols DA et al (1988): Pharmacokinetics of a 5fluorouracil liposomal delivery system. Br J Ophthalmol. 72(9):688-91.

Singh K, Bhattacharyya M, Mutreja A, Dangda S (2018): Trabeculectomy with subconjunctival collagen implant in Indian eyes: Long-term results. Indian J Ophthalmol. 66(10):1429-34.

Skuta GL, Parrish RK (1987): Wound healing in glaucoma filtering surgery. Surv Ophthalmol. 32:149-70.

Soohoo J, Seibold L, Laing A, Kahook M (2012): Bleb morphology and histology in a rabbit model of glaucoma filtration surgery using Ozurdex or mitomycin-C. Mol Vis.

\section{8:714-9.}

Takai Y, Tanito M, Ohira A (2012): Multiplex cytokine analysis of aqueous humor in eyes with primary open-angle glaucoma, exfoliation glaucoma and cataract. IOVS. 53:241-7.

Takeuchi K, Nakazawa M, Ebina Y et al (2010): Inhibitory effects of trehalose on fibroblast proliferation and implications for ocular surgery. Exp Eye Res. 91(5):567-77. 
Takeuchi K, Nakazawa M, Yamzaki H et al (2009): Solid hyaluronic acid film and the prevention of postoperative fibrous scar formation in experimental animal eyes. Arch Ophthalmol. 127(4):460-4.

Tan YF, Mundargi RC, Chen MH et al (2014): Layer-by-layer nanoparticles as an efficient siRNA delivery vehicle for SPARC silencing. Small. 10(9):1790-8.

Tham YC, Li X, Wong TY et al (2014): Global prevalence of glaucoma and projections of glaucoma burden through 2040: a systematic review and meta-analysis. Ophthalmology. 121(11):2081-90.

The Fluorouracil Filtering Surgery Study Group (1989): Fluorouracil filtering surgery study one-year follow-up. Am J Ophthalmol. 108: 625- 633.

Tilleul P, Denis P, Maignen F et al (1997): Effects of different formulations of mitoxantrone (solutions, nanospheres, liposomes) on glaucoma surgery in rabbits. Ophthalmic Res. 29(4):218-26.

Van Bergen T, Vandewalle E, Van de Veire S, et al (2011): The role of different VEGF isoforms in scar formation after glaucoma filtration surgery. Exp Eye Res. 93(5):689-99.

Van Bergen T, Vandewalle E, Moons L, Stalmans I (2015): Complementary effects of bevacizumab and MMC in the improvement of surgical outcome after glaucoma filtration surgery. Acta Ophthalmol. 93(7):667-78

Wang W, Zhao J, Yang G, Cheng G, Yang X (2014): Heat shock protein 47 is upregulated in conjunctival bleb scarring after filtration surgery in rats. Graefes Arch Clin Exp Ophthalmol. 253(1):91-7.

Wells AP, Cordeiro MF, Bunce C, Khaw PT (2003): Cystic bleb formation and related complications in limbus- versus fornix-based conjunctival flaps in pediatric and young adult trabeculectomy with mitomycin C. Ophthalmology. 110:2192-7

Wells A, Ashraff M, Hall R, Purdie G (2006): Comparison of two clinical bleb grading systems. Ophthalmology. 113(1):77-83.

Wilkins M, Indar A, Wormald R (2005): Intraoperative mitomycin C for glaucoma surgery. Cochrane Database Syst Rev. 4: CD002897. 
Wong TT, Mead AL, Khaw PT (2005): Prolonged antiscarring effects of ilomastat and MMC after experimental glaucoma filtration surgery. IOVS. 46(6):2018-22.

Yamanaka O, Saika S, Ohnishi Y et al (2007): Inhibition of p38MAP kinase suppresses fibrogenic reaction in conjunctiva in mice. Mol Vis. 13:1730-9.

Ye H, Qian Y, Lin M et al (2010): Cationic nano-copolymers mediated IKKB targeting siRNA to modulate wound healing in a monkey model of glaucoma filtration surgery. Mol Vis. 16:2502-10.

Yu D-Y, Morgan WH, Sun X et al (2009): The critical role of the conjunctiva in glaucoma filtration surgery. Progress in Retinal and Eye Research. 30:3-28.

Yu-Wai-Man C, Khaw PT (2014): Developing novel anti-fibrotic therapeutics to modulate post-surgical wound healing in glaucoma: big potential for small molecules. Expert Reviews in Ophthalmology. 10(1):65-76.

Zhong H, Sun G, Lin X, Wu K, Yu M (2011): Evaluation of pirfenidone as a new 4 postoperative antiscarring agent in experimental glaucoma surgery. IOVS. 52(6):3136-42.

Zhu X, Xu D, Zhu X et al (2015): Evaluation of chitosan/aptamer targeting TGF-beta 6 receptor II thermos-sensitive gel for scarring in rat glaucoma filtration surgery. IOVS. 56(9):5465-76.

Figure 1. Use of anterior segment optical coherence tomography (ASOCT) and in vivo confocal microscopy in evaluating bleb structure and function. Panel A displays the appearance of the bleb following surgery in a murine model of glaucoma filtration surgery with application of saline (control) mitomycin $\mathrm{C}$ (MMC) or a novel monocyte chemoattractant protein receptor inhibitor (MCP-Ri). Panel B shows cross-sectional imaging of the bleb with ASOCT that enables quantification of bleb height (dashed lines). Panel C shows the use of in vivo confocal microscopy to demonstrate subepithelial connective tissue spaces that are associated with drainage. 


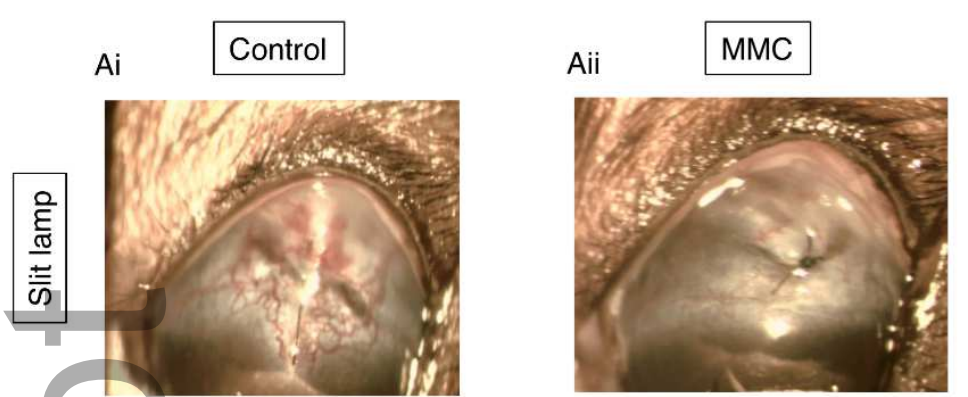

Bii
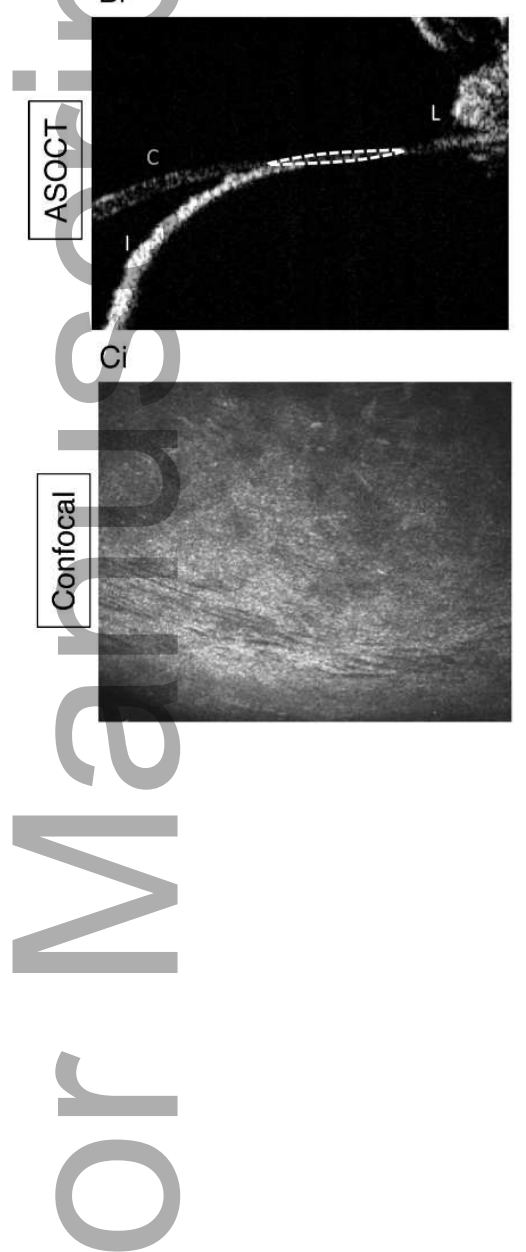



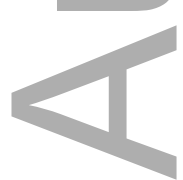

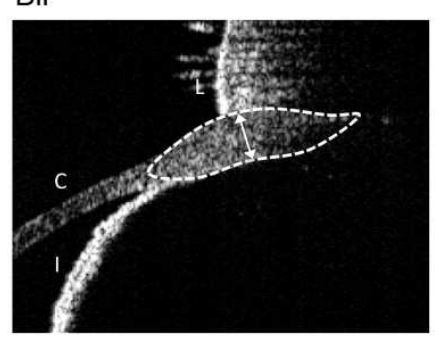

Cii

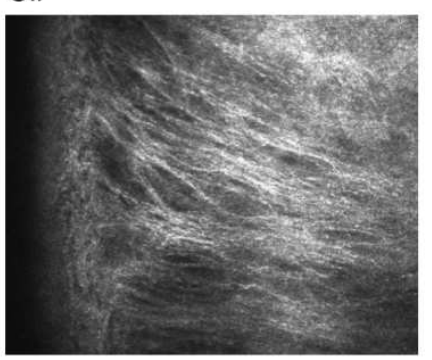

Ciii

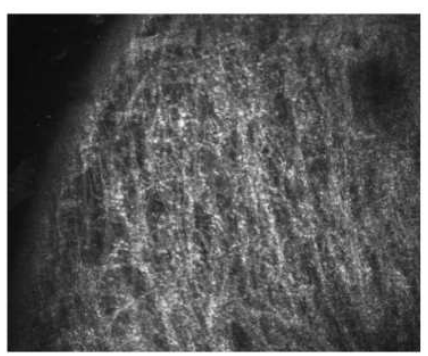

Biii

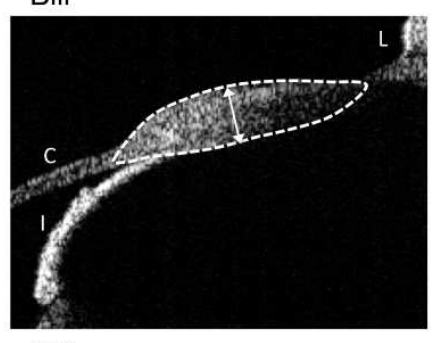




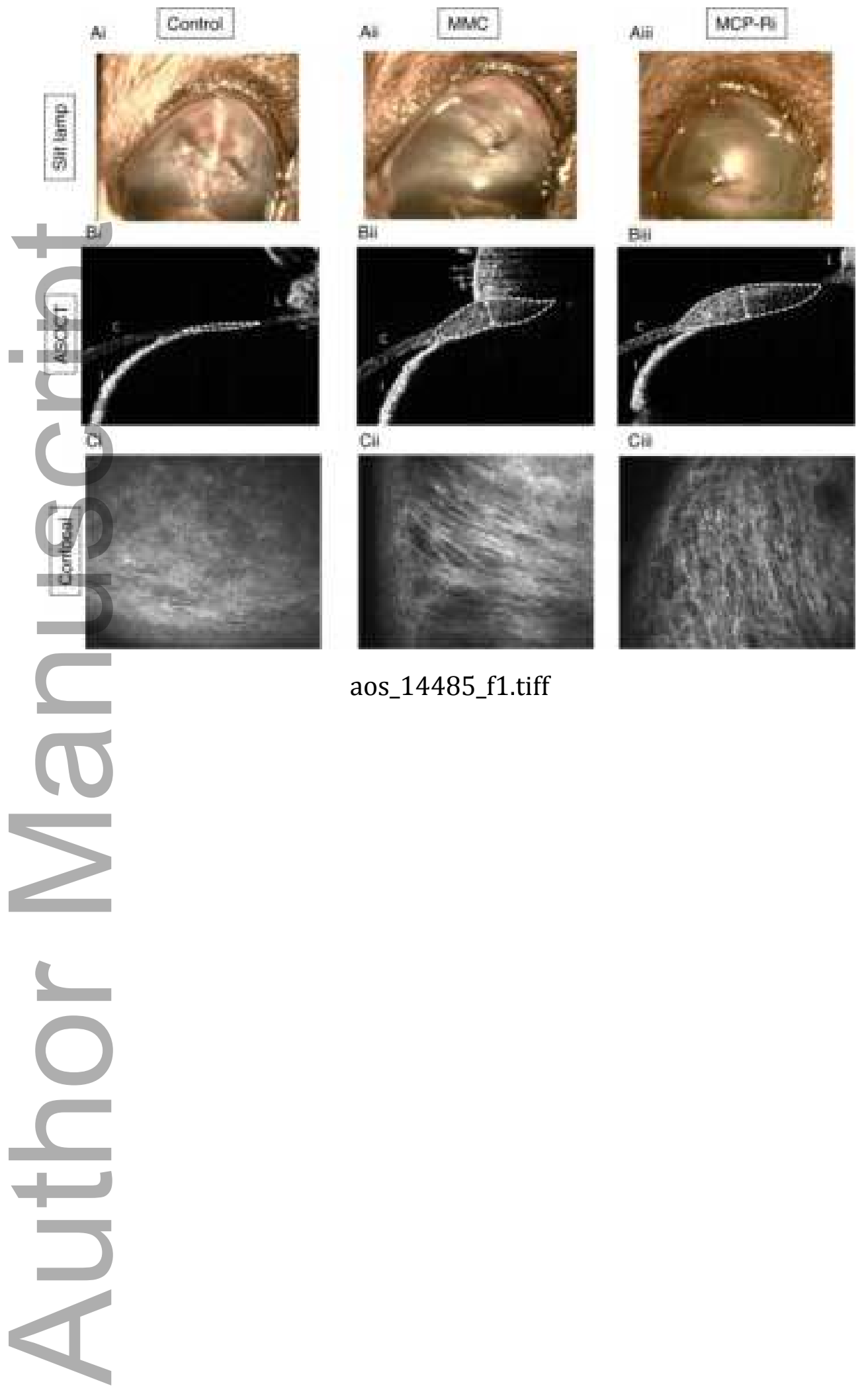




\section{University Library}

\section{- M M I E R R A A gateway to Melbourne's research publications}

Minerva Access is the Institutional Repository of The University of Melbourne

Author/s:

Chong, RS;Crowston, JG;Wong, TT

Title:

Experimental models of glaucoma filtration surgery.

Date:

2021-02

Citation:

Chong, R. S., Crowston, J. G. \& Wong, T. T. (2021). Experimental models of glaucoma filtration surgery.. Acta Ophthalmol, 99 (1), pp.9-15. https://doi.org/10.1111/aos.14485.

Persistent Link:

http://hdl.handle.net/11343/276057 